\title{
Violencia y desplazamiento: Hacia una interpretación de carácter regional y local. El caso de Risaralda y su capital Pereira
}

\author{
Luis Adolfo Martínez Herrera*
}

\begin{abstract}
Resumen. En la presente disertación se realiza un análisis de los fenómenos de violencia que se experimentan en el departamento de Risaralda y su capital Pereira. La reflexión planteada se construye a la luz de una mirada histórica, una caracterización de la violencia y algunas de sus explicaciones causales, una reflexión realizada tomando como referencia el territorio y sus múltiples escenarios que conjugan cruces de actores, intereses y dominios territoriales, un proceso migratorio por violencia que materializa las secuelas del conflicto y una mirada local que reconoce en las sociedades globalizadas, nuevos escenarios de conflictos no regulados por el Estado-Nación. El análisis realizado, permite reconocer las lógicas territoriales de control y dominio, conjuga explicaciones estructurales que manifiestan continuidades y discontinuidades que afectan de manera diferenciada a la población del departamento. La reflexión permite reconocer nuevas preguntas que podrían orientar futuras investigaciones en la región.

Palabras clave: Violencia, historia, desplazamiento, conflicto armado, redes de poder.
\end{abstract}

\begin{abstract}
This dissertation tries to make an analysis about phenomenons of violence in the region of Risaralda and your capital Pereira. The established reflection is made by means of a historical view, a violence characterization and some of your causal explanations, a reflection where the main reference is the territory and your manifold scenes that conjugate actors crossing, interests and territorial dominions, besides, a migratory process by violence (displacement by violence) that materializes the sequels of conflict, and finally, by means of a local view that recognizes in the globalized societies, new non regulated scenes of conflict by the state-nation. This analysis permits to recognize the territorial topics of control and dominion and it identifies some of the structural explanations that show continuities and discontinuities that have an effect on population of the region in a differentiated way. The reflection provokes new questions to guide next investigations in the region.

Keywords: Violence, displacement, Colombian armed conflict, History, power nets.
\end{abstract}

* Profesor de la Universidad Católica Popular de Risaralda, Colombia. Correo electrónico: adomarti@hotmail.com 
El intento por desentrañar la naturaleza misma de la violencia experimentada en el Departamento de Risaralda y en su capital Pereira, exige adoptar una mirada que logre emplear una especie de caleidoscopio con el cual acercarnos al fenómeno, integrando distintas lecturas del escenario interpretado.

De esta manera, en la presente disertación se intentan construir escenarios con los cuales se pueda recrear una mirada más integral de los fenómenos relacionados con la violencia, sus actores, secuelas, cruces y algunas de sus implicaciones en el contexto regional y local. Este proceso de indagación no sólo nutre la mirada respecto al escenario local y regional, contexto que deseamos comprender para poder afectarlo, también permite enriquecer la reflexión acerca de la violencia que experimentamos en Colombia, país que ostenta la tasa de homicidios más alta del mundo y que necesita el desarrollo de interpretaciones regionales, locales e incluso zonales para dar cuenta de un fenómeno rico en matices, cruces y variaciones, situación que hace complejo el panorama a la hora de querer explicarlo y comprenderlo.

Los escenarios por desarrollar son los siguientes: 1) consideraciones generales acerca de la violencia en Colombia y sus manifestaciones en una de las variables más dramáticas del conflicto como lo es el desplazamiento por violencia; 2) mirada histórica del fenómeno de violencia; 3) lectura regional del fenómeno asumiendo una disertación que dé cuenta de los tejidos interregionales de la violencia que afectan al Departamento; 4) una mirada local del fenómeno, sus manifestaciones, actores y algunas de sus secuelas en la ciudad de Pereira, 5) consideraciones finales.

Consiente de que el presente ejercicio no constituye un punto de llegada, sí representa un acumulado que permitirá seguir ahondando futuras reflexiones que nos permitirán reconocer cada vez más, un fenómeno que cambia constantemente sus manifestaciones y sus implicaciones, en una sociedad que se niega a aceptar pasivamente la tesis de una violencia cultural, y que por el contrario, pretende construir lecturas con las cuales se pueda comprender la naturaleza misma de nuestros conflictos y propenda por soluciones negociadas a la compleja y confusa condición humana. 


\section{Escenario 1: Consideraciones generales acerca de la violencia y su manifestación en el desplazamiento forzoso en Colombia}

Comprender las dinámicas propias de la violencia actual en Colombia, exige reconocer las pretéritas interpretaciones realizadas en nuestro país para explicar los fenómenos de violencia que hemos experimentado. En este sentido, en la ponencia de Daniel Pecaut (1993) "Una interpretación global de la violencia", el autor señala cómo en el balance global acerca de las interpretaciones anteriores en Colombia para explicar los fenómenos de violencia, podemos encontrar tres tipos de interpretaciones: 1)ausencia o precariedad del Estado; 2) existencia de una población importante por fuera de la institucionalidad; y 3) compleja situación entre centro y periferia.

En relación con la primer interpretación, él menciona algunas de las confusiones más comunes cuando se hace alusión a la precariedad del Estado, ya que no es sólo su ausencia, como realmente ocurre en algunas zonas del territorio nacional, sino también su presencia con marcadas debilidades:

* Existencia de un Estado con una precaria capacidad para formar una imagen de Unidad Nacional.

* Escasa capacidad del Estado para regular los conflictos sociales.

* Mezcla en el Estado de intereses privados y públicos.

* Un Estado que necesita apoyar su autoridad recurriendo al uso de la fuerza.

A pesar de ello, el autor resalta algunos de los avances realizados en la materia para contrarrestar dichas debilidades; algunos de ellos están relacionados con los esfuerzos para reconstruir el sistema judicial, el interés de atacar internacionalmente el narcotráfico, la modernización de las fuerzas militares y la vocación por aumentar la presencia del Estado en algunas zonas periféricas del territorio nacional.

Aún es necesario avanzar en la capacidad del Estado para regular el conflicto social (existen pocos actores sociales consolidados y 
pocos conflictos sociales organizados) y construir dinámicas de autoridad que contrarresten los niveles de desconfianza existentes por algunos sectores poblacionales hacia la fuerza pública.

Frente a la explicación que señala la existencia de una importante población por fuera de la institucionalidad, el autor retoma la tesis de Fernán González quien afirmaba que este hecho hacía parte de los elementos de larga duración que configuran nuestra violencia de la historia Nacional.

Desde el siglo XVIII y XIX una importante franja de población escapa al control de las autoridades y configuran la costumbre de vivir por fuera de la ley, creando reglas locales y garantizando formas de coexistencia.

En relación con la tercer explicación que señala la tensa situación centro-periferia, Pecaut afirma que dicha situación tiende a fortalecerse en lugar de desaparecer. Al respecto, señala dos causas fundamentales para que dicha dinámica se siga presentando:

La consolidación y explotación de nuevas riquezas (legales e ilegales) en las zonas periféricas y los procesos de descentralización del Estado; ambos factores constituyen escenarios para el desencadenamiento de futuros conflictos detonados por tales situaciones. De esta manera, los efectos de la fragmentación permanente seguirán jugando un papel importante para entender nuestra violencia.

De igual manera, Daniel Pecaut agrega tres elementos que permiten comprender la dinámica nacional de la violencia actual en Colombia. En primer lugar, define cómo la constitución y la permanencia de algunos actores de la violencia se genera a partir del interés por poseer el control de los recursos económicos. Si la característica del conflicto en Colombia en los años cuarenta fue el conflicto bipartidista, en los años setenta la inserción del narcotráfico a finales de los ochenta la guerra sucia, en la violencia actual (denominada como anómica o violencia ordinaria) la disputa por los recursos económicos juega un papel central para la instauración de los conflictos. Las disputas por los cultivos de coca, las zonas petroleras, el oro, las esmeraldas y últimamente el café, son una muestra clara de ello. 
En una segunda instancia, señala la constitución de algunos de los actores del conflicto, en redes de poder. Dicha categorización permite la utilización de un concepto neutral para designar actores legales o ilegales, armados o no, que a través de una dosis de coacción y con referencia a una racionalidad instrumental, llegan a ejercer control económico en algunos sectores de la población y a poseer generalmente una base territorial más o menos definida.

La realización de tal análisis permite reconocer en zonas con vacío de poder (ocasionados generalmente por violencia), la presencia de nuevos actores que empiezan a regular las relaciones y los conflictos sociales.

Frente a estas redes de poder que logran a través de la coacción posicionar un interés común, existe una relación instrumental de parte de las comunidades sobre las cuales estas redes operan. Igualmente en estos escenarios se debilitan las organizaciones sociales y los espacios públicos para el debate, la expresión, el encuentro y la convivencia. Ello no quiere decir que en algunas ocasiones el papel de un Estado paralelo ocasionado por la ausencia real del Estado legalmente constituido, sea asumido por actores ilegales que poseen legitimidad al interior de las comunidades en las cuales éstos operan.

Por último, el autor comenta en su artículo titulado: "Colombia: violencia y democracia" (Pecaut, 1991), la sorprendente capacidad del Sistema Político Colombiano para adaptarse a la violencia, situación que plantea una paradoja en el caso colombiano: frente a la sensación de estabilidad de la sociedad y el estado Colombiano, la tradición civilista, el relativo crecimiento económico y el monopolio de los partidos tradicionales (panorama que ha venido cambiando en las últimas elecciones 2002-2006 en Colombia y que dibujarían un panorama distinto al esbosado por Daniel Pecaut), “¿cómo comprender la combinación de procedimientos ampliamente democráticos y de fenómenos de violencia a la vez explícitos y difusos?" (Pecaut, 1991: 27).

Esta extraña convivencia de guerra y política y la idea difundida en amplios sectores de la sociedad colombiana de que la política y las luchas sociales no pueden ser mediadas por el sistema de 
regulación democrática, si no pasan por el uso de la fuerza. Tal situación favorece cierta normalidad y coexistencia con la violencia.

En este panorama nacional, surge la siguiente interrogante ¿cómo podemos entender las secuelas de la violencia experimentada en Colombia, a partir de los procesos de migración forzosa que en los últimos 10 años (según cifras oficiales) han desplazado a 2.300 .000 habitantes de sus territorios?

Para realizar tal análisis, se presenta a continuación un abordaje genérico del problema migratorio por violencia en Colombia, haciendo énfasis en el tipo de enfoques que han abordado tal problemática.

Entender el conflicto social y armado en Colombia, no puede estar al margen de los signos que lo evidencian. El desplazamiento materializa una de las secuelas del conflicto, representa uno de los más preocupantes fenómenos humanitarios que actualmente experimentamos.

\section{Desplazamiento por violencia en Colombia}

El creciente interés de los estamentos públicos y las ONG's nacionales e internacionales en el país, manifiesta la preocupación creciente de la sociedad sobre la migración por violencia ${ }^{1}$ y sus secuelas; éstas alcanzan dimensiones de catástrofe social, situación que no es para menos si identificamos las cifras $^{2}$ que coinciden en

\footnotetext{
${ }^{1}$ Alejandro Castillejo (2000) caracteriza a la persona desplazada como un sujeto liminal partiendo del concepto elaborado por Víctor Turner quien define la liminalidad como "el estado de ambigüedad o la posición en donde se está al margen de un orden establecido". De esta manera el sujeto se define en transición, carente de las estabilidades que poseía en el pasado, se enfrentan a la contingencia del presente y las incertidumbres por el futuro.

${ }^{2}$ Existen en el país cuatro fuentes nacionales de información - CODHES, Red de Solidaridad, Pastoral Social y CICR - y al menos tres sistemas de información cada uno con sus propios enfoques, intereses, metodologías y recursos, de esta manera existe el a) Sistema de estimación cuantitativa a través de procesos de monitoreo y contrastación de fuentes (RSS y CODHES), b)Sistema de Registro a la Oferta y la Demanda (RSS, CICIR y RUT), c) el Sistema de Caracterización Sociodemográfica y d) Sistemas Cuanticualitativos (Suárez, 2001).
} 
señalar el crecimiento permanente en los últimos quince años del desplazamiento por violencia. En este periodo, tres millones de colombianos han abandonado sus tierras y se han refugiado en condiciones precarias en medianas y grandes ciudades. Colombia pertenece así, al grupo de siete países al lado de Sri Lanka, Afganistán, Sudan, Líbano, Nigeria y Guatemala (figura 1) que concentran la mayor cantidad de personas desplazadas internas por violencia en el mundo entre las cuarenta naciones que afrontan el problema de $24^{\prime} 000.000$ de desplazados dentro de sus territorios (Gaparini, 1998). Por la dinámica del conflicto político, social y armado en el país, se prevén nuevos desplazamientos, como lo señalara Daniel Pecaut (1999): "todo hace pensar que los desplazamientos van a continuar."

Aunque el debate público sobre el desplazamiento en Colombia se inicia a finales de los ochenta como resultado, entre otras razones, de los programas promovidos por Naciones Unidas para fortalecer los procesos de paz iniciados en Centro América, Colombia ya poseía algunos insumos analíticos que intentaban dar cuenta del fenómeno y sus implicaciones más allá de los estudios generales sobre la dinámica migratoria en el país - transición demográfica- con sus cifras, consolidados y proyecciones. Los efectos políticos del proceso de despojo a que han estado sometidos vastos sectores del territorio nacional (Suárez, 2001), han sido interpretados bajo distintas perspectivas: el desplazamiento territorial involuntario (Fajardo, 2001:42), las fronteras abiertas y las frontera cerradas (Tovar, 1980), la frontera agrícola, la frontera de la hacienda, los arrendatarios de frontera, la frontera económica, la frontera jurídica, la dispersión agraria (Bejarano, 1983), perspectivas que además permitieron realizar "un seguimiento al silencio histórico sobre prácticas de dominio territorial que hoy vuelven a emerger para fragmentar la convivencia social" (Suárez y Henao, 2001:17).

Iniciada la década de los noventa, emerge la discusión jurídica basada en el discurso del Instituto Interamericano de Derechos Humanos, y que al referirse a los primeros estudios (Reyes y Bejarano, 1991; ASCODAS, 1991), orientaba las discusiones acerca del despla- 


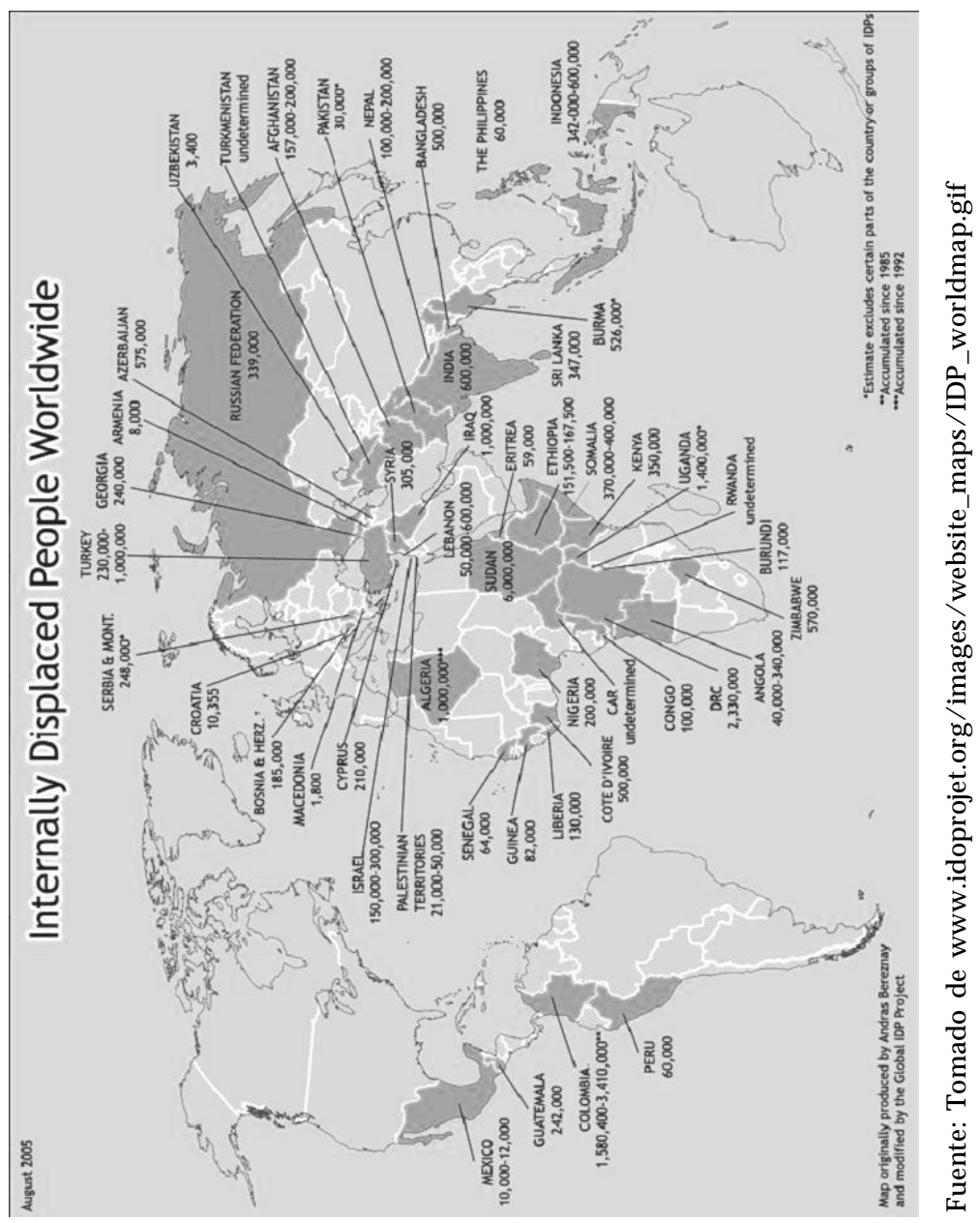

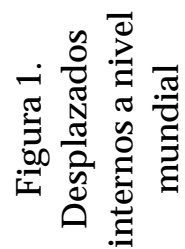


zamiento por violencia. Una mirada esquemática (del conjunto de análisis desde las ciencias sociales hasta 1992), permite argüir que "lograron densificar el análisis sobre la multidimencionalidad de la violencia en Colombia, ampliar el debate de acuerdo con las especificidades locales y regionales de la guerra, elaborar las primeras cifras y ponderaciones, así como la necesidad del monitoreo constante sobre la situación de los Derechos Humanos, y el Derecho Internacional Humanitario." (Suárez y Henao, 2002:22).

Como consecuencia de dichos debates y acompañado de la presión internacional, el desplazamiento por violencia adquiere mayor atención. Se constituye una Política Pública para enfrentar el problema. Luego la realización y publicación de la investigación de la Conferencia Episcopal, ${ }^{3}$ significa un nuevo momento respecto a los estudios que interpretaban el desplazamiento por violencia. Este documento a su vez posibilita los primeros pasos para la constitución de sistemas de información confiables que dan cuenta de la magnitud demográfica del desplazamiento en el territorio Nacional.

Después nuevos estudios cualitativos han iluminado temáticas no analizadas del fenómeno, ubicando su atención en el sujeto que afronta el proceso de desplazamiento en los nuevos escenarios construidos por el conflicto social y armado que afronta el país. De esta manera, Daniel Pecaut define a partir de las nuevas lógicas del conflicto armado (caracterizado por las desterritorialización del accionar de los grupos irregulares, la desubjetivación experimentada por algunos de sus actores y la destemporalización que define nuevos escenarios espacio-temporales vivenciados por las víctimas) (Pecaut, 1999:8-35) las continuidades y discontinuidades experimentadas en los procesos de desplazamiento afrontados en la actualidad respecto a la violencia de los años cincuenta (Pecaut, 1997); también Nora Segura (2000) hace énfasis en la importancia de los estudios que analicen con detenimiento, el comportamiento de las variables género

3 "Derechos humanos: Desplazados por violencia en Colombia", estudio exploratorio realizado por la Conferencia Episcopal Colombiana (1995). 
y generación, vectores que actúan como factores selectivos de expulsión territorial y median los impactos durante el proceso de desplazamiento y las secuelas que éste ha generado, igualmente señala la importancia de pensar el desplazamiento por referencia a la construcción del mundo cotidiano y a la reedición de un proyecto de vida; luego Alejandro Castillejo (2000) define una antropología de la guerra en la que reconoce la insuficiencia de modelos analíticos y metodológicos utilizados para dar cuenta de un fenómeno inestable, transitorio por su propia naturaleza; las lógicas de alteridad ante un sujeto liminal como lo es el desplazado, exige un ejercicio de constante reflexibilidad por parte del investigador y su estudio.

Tal proceso de análisis es confrontado con las dinámicas propias del conflicto. El territorio lo configuran las tensiones de poder en las cuales los grupos irregulares disputan la capacidad de coacción sobre las poblaciones en las que ejercen dominio y los recursos que les permitan continuar con sus objetivos trazados. En las figuras $1 \mathrm{y}$ 2 se ubican los impactos del desplazamiento en Colombia.

\section{Escenario 2. Contextualización histórica}

Una mirada a la historia de laviolencia en Colombia permite reconocer los distintos tipos, manifestaciones y características de un fenómeno que cambia permanentemente en el tiempo, pero que recrea la sensación de continuidad, evidencia algunas secuelas que al parecer trascienden en el tiempo y se incorporan en la memoria colectiva como un signo distintivo de nuestra tradición y nuestra cultura. Ya hemos mencionado algunos de los peligros propios de tales tesis, ahora se desea realizar una mirada histórica que nos permita configurar un contexto con el cual entender la violencia que nos acosa en la actualidad.

La mirada de larga duración remite a realizar una breve caracterización acerca del Estado-Nación en Colombia, factor que ha sido señalado como una de las explicaciones clásicas para entender la violencia en nuestro país. 


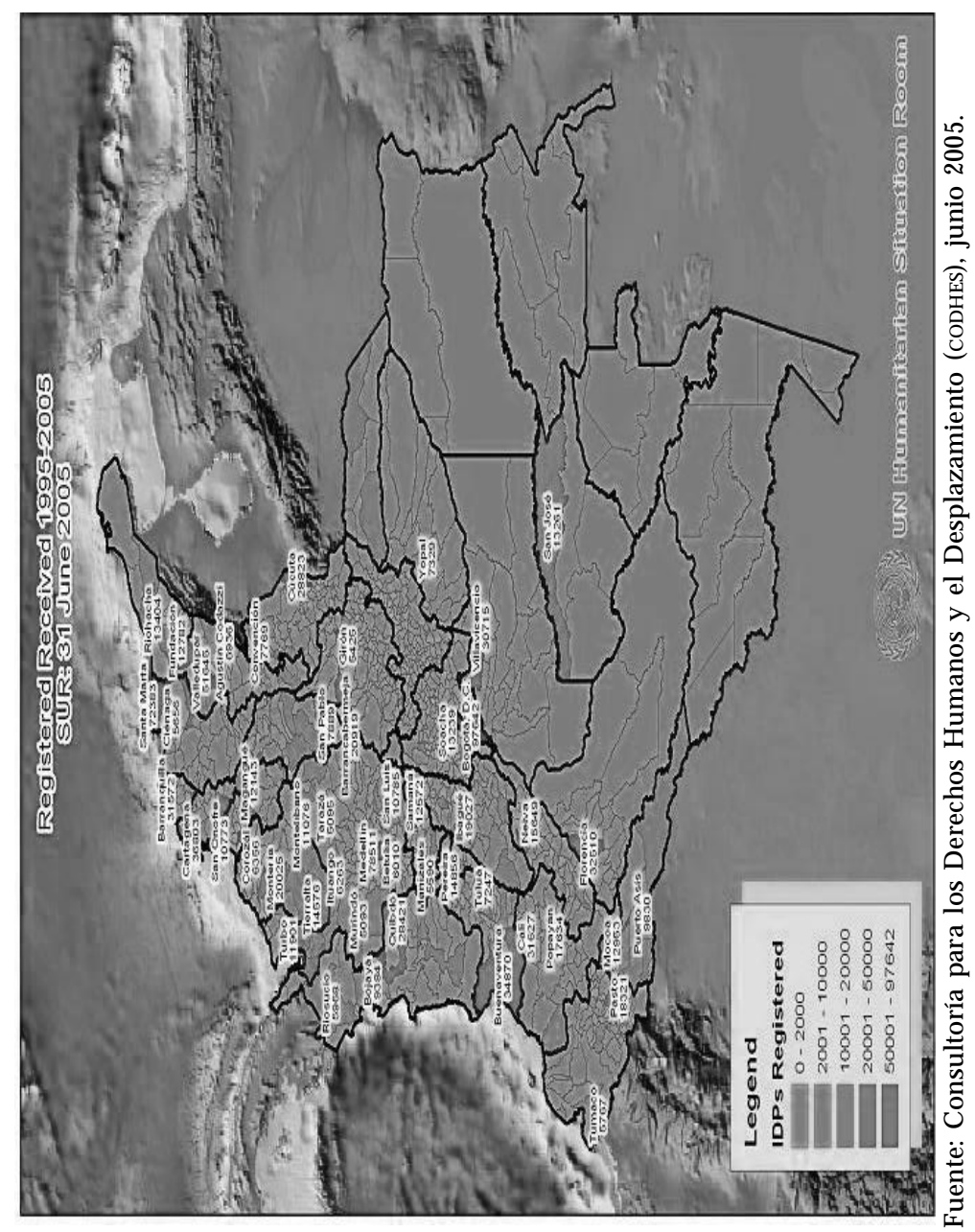

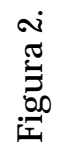




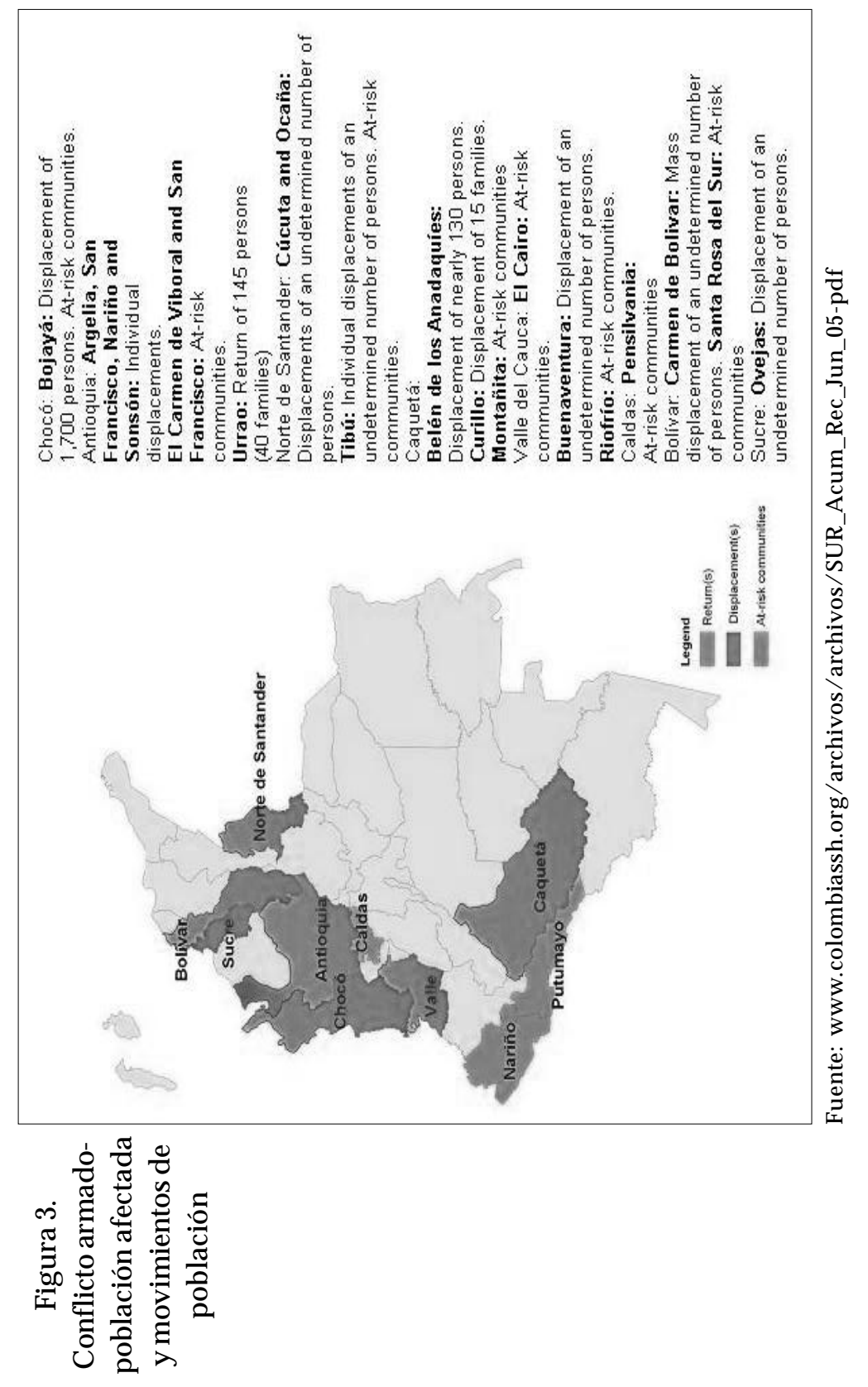


Si el papel originario del Estado-Nación, (concebido desde la modernidad y no necesariamente desde el mundo contemporáneo, factor por analizar en próximas disertaciones que ubiquen el interés en la llamada modernidad tardía, reflexiva o como diría U. Beck sociedades del riesgo) era el de homogenizar el poder y el control en un territorio que articulara una cultura, una economía y unas instituciones, el Estado colombiano no logró configurar una simbología de unidad nacional. Al respecto, Pecaut señala la incapacidad del Estado para recrear un simbolismo social ocasionado fundamentalmente (en un momento particular de la historia) por la división del territorio nacional propiciado por los partidos políticos. Esta situación limitó la construcción de una mirada unificada de la nación. Este fraccionamiento espacial del territorio en poderes locales, estuvo acompañado de la incapacidad del Estado para construir mecanismos constantes de regulación social. El estado colombiano no pudo configurar un simbolismo económico, por el contrario lo que primó por ejemplo en la crisis cafetera a mediados del siglo $X X$, fue la tesis del Laisser faire el — dejar hacer- que define una no intervención del Estado en el juego de la libre competencia y las economías de mercado. A su vez, tales dinámicas de precariedad del Estado estuvieron fortalecidas por el clientelismo que impidió la modernización de las instituciones.

Pero tales condiciones relacionadas con el Estado-Nación, no logran agotar la mirada a la historia de la violencia (y mucho menos explicarla), de igual modo las explicaciones que limitan las causas de la violencia a factores estrictamente políticos o a las condiciones de miseria. Es necesario reconocer algunas particularidades que definan puntos de encuentro entre los académicos que han interpretado los fenómenos de violencia en nuestro país. Al respecto, tres premisas consensuadas desde la academia orientan a continuación la mirada a la historia de la violencia: la inexistencia de un momento originario de la violencia en Colombia; la discontinuidad que matiza las distintas violencias hasta ahora experimentadas y; la imposibilidad de señalar límites entre los actores, intereses, formas y usos de las múltiples violencias (por 
ejemplo los límites existentes entre la violencia social y política y la violencia ordinaria). ${ }^{4}$

Frente a la primer afirmación, no podemos ubicar un inicio de la violencia en el siglo XIX (en la guerra de independencia a principios de siglo, en las sucesivas guerra civiles regionales o internacionales, o la guerra de los mil días a fines del mismo siglo), tampoco en las luchas agrarias de 1920 a 1935, o en la violencia bipartidista exasperada en 1948 con la muerte de Gaitán y el desplome de un proyecto político de nación, tampoco en la guerra sucia o la violencia política en las años ochenta.

En relación con la segunda afirmación, los procesos de discontinuidad no sólo se dan entre una época (o pico alto de violencia) y otra, también se generan a partir de las realidades sociales en donde se manifiestan los fenómenos de violencia: "los diversos estudios que conocemos confirman un hecho: la diversidad extraordinaria de los fenómenos de violencia [...] de un departamento a otro, de un municipio a otro, de una vereda a otra, las luchas bipartidistas, los conflictos sociales, y el vandalismo se combinan y se organizan de maneras diferentes alrededor de una multiplicidad de protagonistas" (Pecaut, 1997:262) Tal situación coloca de manifiesto la tercer premisa que ubica los límites confusos entre una violencia y la otra.

Los cruces de intereses, las formas de organización territoriales que particularizan tácitos acuerdos entre los actores y las dinámicas diversas que adquieren las violencias actuales en lo que Daniel Pecaut ha llamado los procesos de desubjetivación de los actores del conflicto social y armado, situación que describe a un sujeto en crisis, panorama que promueve la deserción e incluso el paso de un actor de un grupo a otro; la desterritorialización que ubica un nuevo escenario del conflicto en el cual un actor atraviesa las fronteras del otro, realiza una acción armada y regresa a su zona de control,

${ }^{4}$ Algunos de los insumos presentados a continuación, fueron recopilados en el análisis que realicé para el Instituto Nacional de Medicina Legal y Ciencias Forenses, regional Risaralda y elaborado en el año 2005. 
descentrando su campo de operaciones y por último, una destemporalización del conflicto que genera una nueva condición de disputa, la cual implica nuevas facetas en la confrontación mediadas por escenarios con acelerados cambios en los procesos de tránsito de información y difusión de la misma.

Tal mirada del contexto de la violencia como dinámica nacional, adquiere vida propia, forma y sentido a raíz de las realidades zonales, locales y regionales que la estructuran desde la base. Un ejemplo de ello lo presenta Germán Castro Caicedo (1976) cuando realizó una radiografía nacional a mediados de los años setenta sobre la violencia en Colombia. Toma como ejemplo representativo de la violencia bipartidista, el caso experimentado en Risaralda en los municipios de Balboa y la Celia. En su texto Colombia amarga (Castro, 1976:9-15) retrata desde el anonimato y la clandestinidad para la construcción de sus crónicas de guerra, la violencia experimentada en estos municipios. "así son Balboa y la Celia, dos pueblos diminutos que se desangraron en la época de la violencia, hace 25 años y que hoy han vuelto a las andanzas porque hay interesados en no dejarlos descansar" (Castro, 1976:15).

Pero ¿de qué manera nos sirve esta mirada histórica para entender la violencia que nos acosa en la actualidad? Para realizar tal análisis deseamos retomar los aportes realizados por Radbruch y Gwinner (1955) quienes evaluaron el impacto de los conflictos prolongados sobre la violencia y las conductas delictivas. Toman como ejemplo los casos experimentados en Francia e Inglaterra. En el primero, posterior a la guerra de los cien años, los distintos historiadores coinciden en señalar cómo dicho conflicto se constituyó en una escuela del crimen para la época. En Inglaterra, por su parte, se señala la continuidad de la criminalidad posterior a las prolongadas experiencias de guerra del siglo XIV (aumento del crimen en las épocas de la tregua).

El caso más representativo de dicha dinámica en Latinoamérica, lo constituye El Salvador, país que posterior a la firma que representaba el proceso de paz en 1992, experimentó un marcado incremento en los índices de criminalidad y violencia homicida. 
Tales ejemplos evidencian una dinámica reiterativa durante la guerra o durante conflictos prolongados, "se legitima el uso de la fuerza y la expropiación de bienes, se difunde la tecnología de las armas, se incrementa el número de personas armadas y se debilita la autoridad civil" (Rubio, 1998).

Aún reconociendo las discontinuidades propias de la violencia en Colombia, la prolongada existencia de un conflicto mediado por el uso de la fuerza, genera la construcción de dispositivos que matizan las relaciones sociales. Tal situación genera la sensación de una violencia prolongada en el tiempo. Es así como las condiciones de la violencia cambian, pero lasdinámicas generadaspor la violencia tocan las prácticas sociales, matizan a los actores y difunden usos y tecnologías propias de las acciones violentas.

De allí que la violencia, sin importar incluso sus móviles, sus causas y sus condiciones, adquiera formas que le permiten una subsistencia normal y no se reconoce como un fenómeno anómico, anormal o desviado. Adquiriendo una corporeidad que se desvanece en la esfera cotidiana.

\section{Escenario 3: mirada interregional}

Antes de caracterizar el territorio, se hace necesario en aras de comprender mejor el presente estudio, presentar un mapa que oriente al lector respecto a la ubicación del departamento de Risaralda en Colombia, y del municipio de Pereira en Risaralda (figura 4).

Configurar el territorio que define las lógicas de la violencia en Risaralda, exige desdibujar la rigidez de las presentes fronteras. Tal panorama permite rastrear los circuitos homicidas que atraviesan al departamento y lo extienden como canal y medio en beneficio de unos intereses definidos.

A continuación se presenta una radiografía de los actores del conflicto en el departamento y su relación con el territorio. Es necesario señalar que la caracterización que se presenta a continuación no significa una diferenciación rígida; como ya se ha señalado, las fronteras entre los actores y sus escenarios no son 
Figura 4.

Ubicación geográfica de Risaralda en Colombia y de Pereida en Risaralda

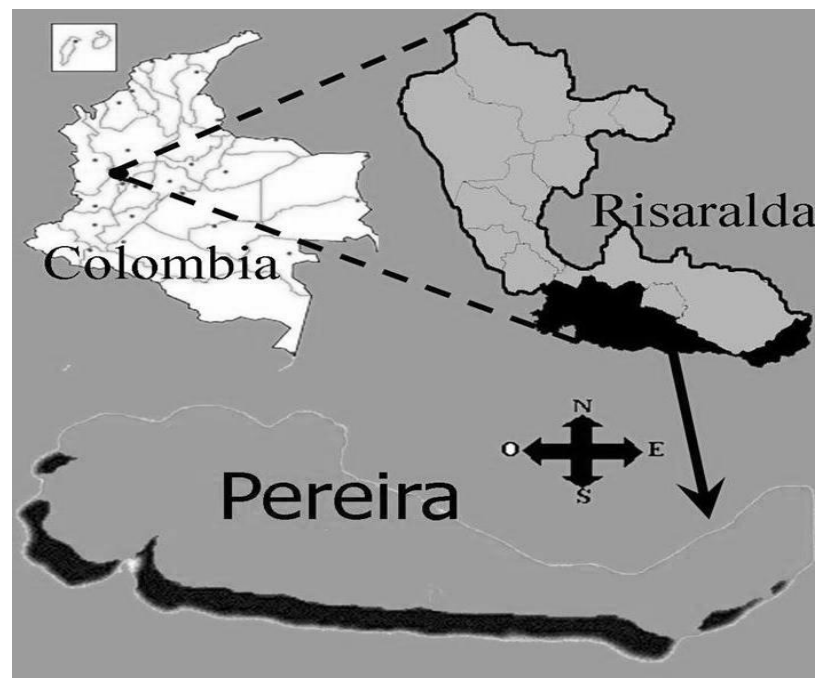

Fuente: Elaboración propia.

siempre excluyentes. De esta manera, el cruce de intereses de un actor influye o dinamiza las acciones del otro, incluso llegando a fusionarse en un escenario determinado.

Territorialidad y el accionar de los principales actores del conflicto armado en la región de Risaralda

El narcotráfico y su creciente presencia en el departamento

Para el año de 1999, los análisis realizados por el Instituto Nacional de Medicina Legal y Ciencias Forenses, señalaban la fuerte influencia del narcotráfico y sus múltiples actores en el departamento de 
Risaralda. Destacaban los elevados índices de homicidio en el norte del Departamento del Valle del Cauca, el sur de Risaralda, y el occidente de Caldas; llamaron tal bloque interregional como el corredor occidental de violencia homicida.

Las bonanzas generadas por el cultivo del café y amapola, la creciente presencia de laboratorios para el tratamiento de sustancias ilícitas y la compra de terrenos por parte de narcotraficantes, provenientes fundamentalmente del Valle del Cauca, generaron escenarios de violencia en los cuales estos nuevos actores ejercían regulación sin la mediación estatal.

De este corredor harían parte los municipios que presentaron los índices más altos de violencia homicida en el año 1999. De esta manera, la Virginia (con una tasa de 227.4 por cada 100.000 habitantes), Marsella (con una tasa de 149.39) y Pereira (tasa de 101), representaban índices (los primeros dos municipios) que los ubicaban a nivel nacional como municipios extremadamente violentos, y que doblaban, o incluso, triplicaban el promedio nacional de violencia homicida. Estas condiciones de violencia exacerbada, propiciaron la conformación de bandas sicariales al servicio del mejor postor en municipios como Marsella y la Virginia, bandas que a su vez, ejercían fuerza para obtener ganancias del nuevo flujo de capital presente en la zona.

\section{Fuerte confrontación por disputa territorial}

En el análisis realizado por el Observatorio del Programa Presidencial de Derechos Humanos y Derecho Internacional Humanitario publicado en marzo de 2005, se señala la existencia de un corredor en disputa que cobijaba el sur de Antioquia, el occidente de Caldas y el norte de Risaralda, la razón, las riquezas presentes en la zona (oro, café y recursos naturales) y la disputa territorial entre las AUC y los movimientos guerrilleros presentes en este territorio (FARC y ELN). ${ }^{5}$

${ }^{5}$ Las FARC (Fuerzas Armadas Revolucionarias de Colombia), constituyen el grupo insurgente, aún vigente, de mayor historia en América Latina. De vocación marxista-leninista, fue creado en 1963, posterior al fracaso militar de 
La presencia histórica de las guerrillas, especialmente de las FARC con el bloque central conformado por los frentes 47, el cual opera en los límites entre Caldas y Choco, el frente 50 que tiene influencia en Santa Rosa y Dosquebradas y la compañía Aurelio Rodríguez ubicada en la zona Norte de Mistrató, Pueblo Rico, Guática y Quinchía; el eln con el frente Cacique Calarca, Ernesto Che Guevara y el núcleo urbano Martha Elena Barón (los cuales operan en Pereira, Pueblo Rico, Mistrató y Belén de Umbría) y el EPL que opera en el viejo Caldas y Antioquia con la disidencia del frente Oscar William Calvo, afrontan el incremento de la confrontación ocasionado por dos razones: las AUC presentes en el departamento en los años ochenta asumen, a nivel nacional a mediados de los años noventa, intensificar su accionar en el territorio Colombiano.

De esta manera, los frentes cacique Pipintá, héroes y mártires de Guática, se fortalecen con los grupos de justicia privada promovidos por el experimento de seguridad denominado para la época como las "Convivir", que operaron en Guática, Belén de Umbría y Pereira.

De igual manera, tales grupos fortalecen su accionar con los vínculos entre dichas organizaciones y los carteles del Valle del Cauca. Las autodefensas se involucran con el tráfico de cocaína liderados por Lorenzo Gonzáles Quinchía alias "macaco" o Javier Montañés, quien a su vez habría comprado tierras y empresas en el territorio cafetero.

Marquetalia (pequeño territorio en el sur del departamento del Tolima), cuando el gobierno intentó retomar una zona olvidada del Estado y que decide revelarse ante el olvido estatal. 90 campesinos sobreviven a la acción militar de gran envergadura y la tradición político militar que dejó la violencia del 50 en Colombia con las guerrillas liberales y su disputa con los militares y los grupos conservadores que auspiciaban los denominados "pájaros" perfilan algunas de las bases para la creación de esta organización guerrillera. Por su parte, la agrupación guerrillera ELN (Ejército de Liberación Nacional), surge en el auge de los movimientos guerrilleros de vocación procubana, con una fuerte influencia de la teología de la liberación en América Latina. Las AUC (Autodefensas Unidas de Colombia), constituyen agrupaciones armadas anticomunistas que surgieron bajo la tutela de comerciantes, narcotraficantes y ganaderos cansados de las "vacunas" —impuestos informales- cobrados por los grupos insurgentes para financiar sus respectivos proyectos y objetivos. 
El segundo motivo que intensifica la confrontación bélica en dicha zona, define el bloque tres que se describe a continuación.

Las FARC y su intención de tomar el corazón del eje cafetero

La crisis del eje cafetero ocasionada por la decisión de Estados Unidos y algunos países centroamericanos de terminar el acuerdo mundial del café en 1989, generó un proceso de deterioro en la ya menguada economía del mediano y pequeño caficultor. Tal situación generó dinámicas de migración económica y promovió las condiciones para el desarrollo de actividades ilícitas y de delincuencia común resquebrajando el tejido social y aumentando los índices de criminalidad en la zona; ante tal panorama, las FARC decidieron, a comienzos de los noventa, "tomarse el corazón del eje cafetero como rechazo a la ruptura del pacto" ${ }^{\prime \prime}$. De igual manera las FARC pretendían crear un corredor que les permitiera, por un lado, asegurar la salida al mar a través de Chocó, contando con los municipios de Pueblo Rico y Mistrató, y facilitar un paso permanente entre el sur y el norte del país. La riqueza natural de dicha zona no sólo se encontraba por la presencia del parque natural Tatama, también por la presencia de minas de oro ubicadas en tales sectores. Obtener el control del corredor que permitiera la circulación entre el Oriente del Choco, el sur de Antioquia, y el occidente de Caldas, le exigió a las FARC fortalecer su influencia en los municipios de Pueblo Rico, Mistrató, Guática, Belén de Umbría y Quinchía, corredor que pretendía romper las AUc en los municipios de Guática, Belén de Umbría y Quinchía.

Pero no solamente los grupos ilegales nutren los índices de homicidios ocurridos en el departamento, las acciones de la fuerza pública de manera directa o indirecta, también han generado condiciones que inciden en la tasa de homicidios de Risaralda. Un caso de ello lo representa Quinchía y la detención masiva que experimentó dicho municipio el 28 de septiembre de 2003.

${ }^{6}$ Texto elaborado por el Observatorio del Programa Presidencial de Derechos Humanos y Derechos Internacionales Humanos (DIH). Marzo de 2005. 
El pasado 28 de septiembre, la unidad de Derechos Humanos de la Fiscalía General de la Nación en conjunto con mil efectivos de la policía nacional, realizaron un operativo en el municipio de Quinchía, en el que allanaron un significativo número de viviendas de pobladores de la región y capturaron a 90 personas entre ellas dos menores de edad, dos hombres que superan los 73 años y un invidente (Velez, 2005). ${ }^{7}$

Al respecto la personera, Maria Isabel Abad, considera incalculable el impacto económico, social y político de la llamada operación "Libertad":

El 90 por ciento de los detenidos son campesinos. Las mujeres tuvieron que asumir como jefes de hogar y ocuparse en oficios varios, se acentuó el hambre, las personas no tenían cómo visitar a sus presos [...] Ante el señalamiento, a mediados del año pasado llegaron los paramilitares y en 15 días mataron a 15 personas; hubo desplazados y el campo dejó de ser productivo. No había compradores y el comercio decayó (Velez, 2005:14).

En contextos de violencia, los procesos de estigmatización, como diría Gofman, operan como marcas sociales que discriminan, propician la exclusión generando en el afectado procesos de impotencia de la cual es víctima. Tales marcas que no sólo vetan personas sino incluso territorios, erigen zonas de peligro, se recrean imaginarios que no sólo provocan reacciones de fuerza por parte de los actores violentos, sino también discursos o comentarios que justifican la calamidad del otro, víctima de una violencia justificada.

De esta manera, Quinchía ocupa el segundo lugar en el 2003 como municipio expulsor de población desplazada por la violencia

7 Comunicado hecho por el equipo Nizkor de defensa de los derechos humanos el 1 de octubre de 2003. Tal comunicado fue tomado del documento elaborado por el Etno-educador Julian Velez, titulado: Proyecto construcción social e institucional de la Justicia en equidad en el eje cafetero colombiano (2005). 
y en el 2004 el primer lugar con 743 personas, que representaron para la época el $44 \%$ del total de personas expulsadasen el Departamento de Risaralda.

El territorio como lugar antropológico define la construcción de sentidos, propicia la acumulación de memoria y tradición, configurando prácticas construidas por sus habitantes.

Los procesos de desterritorialización del conflicto redefinen las fronteras y las estrategias nacionales de los actores armados; atraviesan las realidades locales; los flujos de lo actores ilegales afectan los escenarios sociales mediando en los conflictos y en las dinámicas cotidianas de las comunidades.

\section{Escenario 4. Lectura acerca de los fenómenos de violencia en la ciudad de Pereira}

Las aceleradas dinámicas de crecimiento experimentadas por la ciudad de Pereira manejan una contradicción que matiza el tipo de crecimiento de la ciudad. Por un lado, las fuertes dinámicas migratorias (fundamentalmente hacia España) son acompañadas por la llegada de comunidades desplazadas por la violencia o por la falta de oportunidades en el campo o en ciudades intermedias estancadas económica y socialmente. A pesar de ello, algunas preguntas empiezan a florecer ¿qué tipo de desarrollo está afrontando la ciudad?, ¿cuáles son los costos de dicho desarrollo?, ¿se están, acaso, modernizando instituciones como la familia, las organizaciones sociales, la cultura política o el Estado?, ¿existe alguna relación entre el tipo de ciudad que estamos construyendo, con los índices de homicidios que experimentamos?, ¿qué nuevos actores y redes dinamizan nuestra violencia?

Pereira, eje estratégico entre Medellín, Cali y Bogotá, experimenta los rigores de múltiples formas de violencia. Mutación de unas dinámicas atravesadas por un mundo globalizado con los vestigios de prácticas tradicionales.

Antony Giddens define la tradición como resultado de un proceso activo y social de reconstrucción permanente del pasado desde el 
presente y el futuro. Un pasado que no existe en su pureza. "como tal la tradición es un hecho producido desde el movimiento de la propia modernidad, está incluido en su dinámica. Hace de la noción de tradición un concepto variable en el tiempo" (Luna, 2000).

Para agudizar tal panorama, el juego de intereses se acopla a las pautas propias de la tradición e incorpora imaginarios que no necesariamente se avalan en la realidad. El slogan —el mundo es tuyo- presente implícitamente en la película El Odio, se desdibuja en una realidad que transforma en una cruel sátira tal idea de sociedad.

Este proceso de doble vínculo enuncia una inclusión coartada en la práctica, ¿no se mata acaso por unos tenis Niké o unos Adidas? Pero tal panorama no sólo se erige como un proceso mediado por las relaciones de clase. Como ejemplo de ello, se puede nombrar el estudio realizado a comienzos del año 2000 en la ciudad de Pereira, en el cual se señalaba la existencia de pandillas conformadas por jóvenes de estratos 5 y 6 dedicados a actividades delictivas. El consumo de drogas, los perfiles de vida fácil, la desarticulación de los núcleos familiares y la influencia de las organizaciones delictivas legales e ilegales, entre otras, constituyen escenarios propicios que justifican y utilizan la violencia como mecanismo de mediación social.

Pero no sólo la violencia se origina por las dinámicas propias estructuradas al interior de la cuidad. Las secuelas del conflicto armado, la falta de oportunidades en el campo y los altos niveles de pobreza, promueven crecientes flujos migratorios hacia la ciudad. De esta manera, Pereira, el triángulo de oro por su ubicación respecto a las ciudades capitales, se constituye en un referente de llegada para las familias que buscan protección o nuevas oportunidades. La dinámica nacional ampliamente ha demostrado cómo las comunidades receptoras de población desplazada, son las comunidades más vulneradas.

A la falta de oportunidades ya existentes en dichos espacios, llegan nuevos actores comunitarios (desplazados por la violencia que llegan con los estigmas propios de la guerra) que entran en disputa por las ya precarias condiciones de subsistencia. Esta 
situación dinamiza conflictos que desarticulan los lazos comunicantes y se forjan solidaridades excluyentes, grupos de interés que entran en disputa por los escasos recursos legales presentes en la zona.

Tal panorama es interpretado por los actores ilegales que obtienen provecho de estas circunstancias y promueven la vinculación fundamentalmente de los jóvenes en sus organizaciones. Ante una ciudad que no los ha incluido para la satisfacción de sus necesidades básicas, encuentran en los grupos ilegales la inclusión que indiscutiblemente necesitan.

De esta manera, son los hombres jóvenes el grupo poblacional más afectado por la violencia homicida, no sólo en Colombia sino también en todo el territorio nacional. Las aún escasas posibilidades de acceso al sistema escolar (aunque se mejoren las condiciones de ingreso, las condiciones reales de sostenibilidad para evitar la deserción escolar, son precarias), las dinámicas de inestabilidad laboral, las dificultades en el acceso a adecuados modelos de salud pública y en general, las condiciones estructurales propias del país, limitan la satisfacción de las necesidades básicas, situación que genera condiciones para la inclusión en las redes ilícitas y promueve escenarios mediados por las acciones de fuerza ante unos modelos de justicia que manejan (según informes propios de la Defensoría del Pueblo) niveles de impunidad cercanos al $97 \%$.

\section{Una mirada hacia las comunas}

Es importante destacar los importantes esfuerzos de instituciones como Medicina Legal para construir los mecanismos que permitan recolectar, procesar y analizar los datos concernientes a los homicidios en Pereira y en la región. Esta información permite avanzar en análisis más sustantivos respecto a la violencia que actualmente experimentamos.

Al realizar una mirada de siete años (desde 1999 hasta el 2004) acerca de la tasa de homicidios por comuna en la ciudad de Pereira, encontramos cómo tres comunas (Comuna Centro, Cuba y Villa Santana) que representan el $23.85 \%$ de la población total del 
municipio, han concentrado el 50\% de los homicidios registrados en la ciudad en estos años.

Casi la tercera parte de los homicidios se registran en la Comuna Centro, la cual alberga el $12.6 \%$ de la población del municipio. En tal zona se concentra una fuerte actividad comercial. Bares, cafeterías y almacenes entre otros, constituyen zonas de encuentro y flujo permanente de personas que colocan en juego un sin número de intereses, muchos de los cuales finalizan con el uso desmedido de la fuerza. En esta zona fluyen los distintos circuitos ilegales los cuales, en algunas ocasiones, chocan con las dinámicas cotidianas de quienes transitan por dichos espacios. Por otro lado,

\begin{abstract}
hablar de una "violencia generalizada" es indicar precisamente que, en la coyuntura actual, elementos de ordenes diferentes tienden a entrar en resonancia y producen consecuencias contradictorias; exacerban una conflictividad difusa y fragilizan a los actores sociales; así mismo suscitan en ciertos casos, una extrema radicalización política y confunden además, lo que pertenece a lo político con lo que no le pertenece (Pecaut, 1991).
\end{abstract}

Tal afirmación refuta las distinciones tajantes entre la "violencia política" y la "violencia ordinaria". Se generan cruces de intereses, medios, actores y escenarios que confunden la una con la otra.

La focalización de la violencia genera en las comunidades afectadas procesos de "adaptación del individuo al entorno predominante" (Norbert, 1994). Tal proceso que configura pautas de ocultamiento, genera un tipo de mimesis que define una seguridad ontológica ante un contexto violento. Esta práctica fomenta la no denuncia, ella a su vez propicia una impunidad que fortalece al victimario y sus acciones futuras.

Es en estos escenarios en los cuales se instauran y se recrean nuevos actores que dinamizan la violencia del municipio. Uno de ellos que genera elevadas prácticas delictivas, culpable de muchos de los homicidios en Pereira, surge como resultado de la desarticulación de los carteles de Cali y Medellín. Algunos de dichos 
actores desplazaron su eje de operaciones y sus amplios recursos al corazón del eje cafetero.

De esta manera surge lo que el Observatorio del Programa Presidencial de Derechos Humanos y Derecho Internacional Humanitario ha denominado "el cartel de Pereira". Bajo su tutela no sólo operan bandas dedicadas a la extorsión y el sicariato, quienes a su vez entran en pugna para beneficiarse de los amplios recursos fluctuantes en el medio. También operan otro tipo de redes ilegales que actúan bajo su control. Apuestas, trata de blancas y prostitución infantil, configuran circuitos que actúan en la ciudad.

En el transcurso de los años 2004 y 2005 fueron asesinados 18 prestamistas, igualmente han caído 33 indigentes y 3 docentes sindicalizados (entre otros sectores sociales), homicidios que explicitan organizaciones que bajo distintos intereses, operan planificadamente en la ciudad.

\section{Consideraciones finales}

Una mirada de larga duración acerca de los fenómenos de violencia en la región, señalan la existencia de factores que definen discontinuidades — viraje de los actores fundamentalmente del narcotráfico hacia el eje cafetero como epicentro de nuevas operaciones-, crecientes vínculos de dichos actores con organizaciones delictivas en la región, consolidación de nuevas redes de poder, caso apuestas en la ciudad, fortalecimiento de un corredor estratégico de las FARC que les permite movilidad desde el Chocó hasta el sur de Antioquia, el occidente de Caldas y el oriente del Tolima, con factores que podríamos considerar de larga duración como ausencia y precariedad del Estado-Nación por ejemplo para solventar y superar la reciente crisis cafetera, los altos niveles en concentración de las riquezas en pocas manos, la ausencia de una imagen nacional que fortalezca la unidad y la identidad nacional al interior del departamento.

Esta situación coloca de manifiesto la creciente consolidación de redes de poder que fragmentan el territorio departamental, por 
ejemplo, la creciente fuerza de redes del narcotráfico en eje norte del Valle del Cauca, sur de Risaralda (fundamentalmente en los municipios de La Virginia, Marsella, Pereira y Santa Rosa) con el oriente de Caldas creando nuevas fronteras en las cuales los diferentes actores se disputan las riquezas legales e ilegales presentes en la zona. Los procesos de desterritorialización señalan la fragilidad de dichos escenarios y campos de actuación de los actores, pero ello no puede desconocer los actores consolidados que se disputan los territorios.

Los procesos de desplazamiento continuarán afectando fundamentalmente a la ciudad de Pereira como ciudad receptora, ya que el fenómeno migratorio en Colombia ha señalado cómo las mayores migraciones se presentan en territorios en disputa, lugares en los cuales no existe una red de poder altamente consolidada en una zona en particular. Risaralda posee además de territorios altamente controlados, nuevas zonas por controlar. Las dinámicas del conflicto a nivel nacional, igualmente manifiestan la tendencia al incremento de la confrontación armada y, por consiguiente, su inevitable impacto en el departamento y en la ciudad de Pereira.

A pesar de las discontinuidades propias de nuestra violencia, el uso continuado de la fuerza como mecanismo de regulación social, ha constituido dispositivos que se instauran en la esfera cotidiana, dinamizada por actores que utilizan tales mecanismos para alcanzar sus objetivos.

Sólo examinando nuestra aguda crisis que maneja condiciones de una situación de guerra y desentrañando el complejo cruce de sentidos, actores y escenarios que configuran nuestras violencias, podremos construir las políticas públicas, las acciones y las movilizaciones sociales a nivel local, regional y nacional, para confrontar tan complejo panorama.

Reconociendo los importantes esfuerzos y adelantos por distintas instituciones públicas para monitorear la violencia homicida en Colombia, aún los análisis para desentrañar los móviles, los actores y los mecanismos causales son precarios. Tal situación limita la realización de análisis más cercanos a la violencia homicida que enfrentamos. 
Los altos niveles de impunidad promueven el exitoso accionar de los violentos, deterioran los hábitos y las costumbres en la esfera cotidiana fracturando el tejido social.

Aún las políticas públicas a nivel local y nacional, no logran frenar lo índices de criminalidad que experimentamos en la actualidad. Más allá de la fluctuación de los indicadores, las tendencias de larga duración se sostienen sin ser realmente afectadas.

Las dinámicas de exclusión que experimentan algunos sectores poblacionales, impactan de manera diferencia a la población afectada; de esta manera variables como las de género y generación definen comportamientos diferenciados que adquieren marcadas diferencias en los niveles de violencia que experimentamos.

De esta manera, los hombres jóvenes e incluso los niños, encuentran en las pandillas lugares incluyentes que confrontan las negaciones instauradas en la familia e incluso la sociedad y construyen espacios identitarios que brindan seguridad y sentido de pertenencia. Las mujeres y en especial las niñas, confinadas en muchas ocasiones a los espacios domésticos, padecen los mayores impactos provenientes de la violencia intrafamiliar y fundamentalmente la violencia sexual. Tal situación favorece la expulsión a una calle que no diferencia la condición generacional.

Se reconoce en la violencia homicida, no sólo factores de carácter estructural (ya sea por las dinámicas propias del conflicto armado o los niveles de desigualdad social que promueven la inclusión al mundo de lo ilícito), sino también elementos de órdenes diferenciados como lo son la inserción a un mundo globalizado, moldeando paradigmas de ser y estar en lo social que seducen e incluyen en el discurso pero que excluyen en la cotidianidad.

Pereira se consolida como epicentro comercial del eje cafetero, se incrementa su actividad turística y se constituye en un foco de desarrollo para la región, pero ¿se está asumiendo el costo social de tal desarrollo?, ¿existen las políticas públicas que puedan prever el inevitable crecimiento que experimentará la ciudad en los próximos años?, ¿estamos construyendo una ciudad incluyente política, social y económicamente dirigida a la gran mayoría de sus habitantes? 
Continuar el camino hacia la indagación cualitativa, se constituye en un acierto que nos permite ir dibujando las dinámicas internas que promueven la violencia. De esta manera, se confronta el accionar de los violentos y se identifican las condiciones que fomentan la acción violenta frente a los conflictos que experimentamos.

Aún así, fenómenos como el desplazamiento por violencia al interior de la misma ciudad (de un barrio a otro) continúan en el anonimato; sin cifras ni estudios que aclaren sus dinámicas y sus impactos en las redes sociales. Estos temas constituyen entre otros posibles estudios por realizar, materia de futuras investigaciones que permitan descifrar aún más el complejo panorama que enfrentamos en la actualidad.

\section{Bibliografía}

ASCODAS (1991), "La nueva colonización urbana. El desplazamiento forzado". Revista Reflexión Política, año 5, núm. 10, diciembre, UNAB, Bogotá, Colombia.

Bejarano, Jesús Antonio (1983), “Campesinado, luchas agrarias e historia social: Notas para un balance historiográfico", Anuario Colombiano de Historia Social y de la Cultura, núm. 11, Universidad Nacional de Colombia, Facultad de Ciencias Humanas, Departamento de Historia, Bogotá, pp 251-304.

Camacho, Álvaro y Guzmán, Álvaro (1990), Colombia, ciudad y violencia, Ediciones Foro Nacional, Bogotá.

Castillejo, Alejandro (2000), Poética de lo otro: Para una antropología de la guerra, la soledad y el exilio interno en Colombia, Instituto Colombiano de Antropología, Bogotá, Colombia.

Castro, Germán (1976), Colombia Amarga, Carlos Valencia Editores, Colombia, pp. 9-15.

Concha y Espinosa (1997), La violencia en Colombia. Dimensiones y políticas de prevención y control, lesiones personales no fatales, Cisalva, Univalle, Cali, Colombia.

Conferencia Episcopal Colombiana (1995), “Derechos humanos: desplazados por violencia en Colombia", Informe de la 
Conferencia Episcopal Colombiana. Santa Fé de Bogotá. Colombia.

Erving, Goffman (1970), Estigma: la identidad deteriorada, Amorrortu Editores, Buenos Aires, Argentina.

Fajardo, Darío (2001), “La tierra y el poder político; la reforma agraria y la reforma rural en Colombia", Ponencia presentada en el Seminario permanente sobre problemas agrarios y rurales: Proyecto viabilidad y reconstrucción de la sociedad rural colombiana, diciembre, Santafé de Bogotá, Colombia.

Gasparini, L. (1998), "El impacto redistributivo del gasto público social. Discusión metodológica y una aplicación al sector educación: Argentina, 1996", mimeo. Dirección Nacional de Programación del Gasto Social, Argentina.

Instituto Nacional de Medicina Legal y Ciencia Forenses. Centro de Referencia Sobre la Violencia (1999), Datos para la vida, Bogotá. Instituto Nacional de Medicina Legal y Ciencia Forenses. Centro de Referencia Sobre la Violencia (2000), Datos para la vida, Bogotá. Instituto Nacional de Medicina Legal y Ciencia Forenses. Centro de Referencia Sobre la Violencia (2001), Datos para la vida, Bogotá. Instituto Nacional de Medicina Legal y Ciencia Forenses. Centro de Referencia Sobre la Violencia (2002), Datos para la vida, Bogotá. Instituto Nacional de Medicina Legal y Ciencia Forenses. Centro de Referencia Sobre la Violencia (2002), Violencia y accidentalidad, Risaralda, Colombia.

Instituto Nacional de Medicina Legal y Ciencia Forenses. Centro de Referencia Sobre la Violencia (2003), Violencia y accidentalidad, Risaralda, Colombia.

Instituto Nacional de Medicina Legal y Ciencia Forenses. Centro de Referencia Sobre la Violencia (2004), Violencia y accidentalidad, Risaralda, Colombia.

Luna, Mario (2000), "Algunas anotaciones sobre el modelo de la sociología clásica". Ponencia presentada en la Universidad de Antioquía los días 2 y 3 de noviembre, Medellín, Antioquía, Colombia.

Norbert, Elías (1994), The civilizing process, Oxford Blackwell. 
(1998)," Ensayo teórico sobre las relaciones entre establecidos y marginados", La civilización de los padres y otros ensayos,

Editorial Norma, Santa Fé de Bogotá, pp. 63-94.

Observatorio del Programa Presidencial de Derechos Humanos y

Derecho Iinternacional Humanitario (2005), Informe sobre los

Derechos Humanos en Colombia en el 2005, marzo, Santa fé de Bogotá, Colombia.

Pécaut, Daniel. (1989), Pasado y presente de la violencia en Colombia.

Centro de Estudios Sobre Movimientos Sociales de París (Cerec),Gonzalo Sánchez y Ricardo Peñaranda (Comp.), Bogotá, pp 267

(1991), "Colombia: violencia y democracia", Revista Análisis Político, núm. 38, mayo- agosto, Luís Alberto Restrepo (traductor), IEPRI, Universidad Nacional.

(1993) "Una interpretación global de la violencia", ponencia presentada en el Banco de la República, Santiago de Cali, Colombia.

(1997). "Análisis de la violencia en Colombia", Revista Estudios

Políticos, núm. 14, enero-junio, Instituto de Estudios Políticos, Universidad de Antioquia, Medellín, Antioquia, Colombia.

(1999), "Configuraciones del espacio, el tiempo y la subjetividad en un contexto de terror: el caso colombiano". Revista Colombiana de Antropología, vol. 35, enero-diciembre, Bogotá, pp. 8-35.

Radbruch, Gustav y Enrique Gwinner (1955), Historia de la criminalidad. Ensayo de una criminología histórica, Bosh, Barcelona.

Rubio, M. (1998), “Crimen con misterio, la calidad de la información sobre la criminalidad y la violencia en Colombia", Reporte de investigación, Centro de estudios sobre desarrollo económico (CEDE), Universidad de los Ándes, Bogotá, Colombia.

Segura, Nora (1998), "Desplazamiento en Colombia: perspectivas de género", Revista Foro, núm. 34, junio, Bogotá.

Suárez, Danilo y Fernando Henao (2002), “Los Eyabidá, la gente de la montaña de Mutatá, Noroccidente de Antioquia", mimeo, Presentado en el diplomado: Política pública y manejo de 
migraciones: el desplazamiento, un reto para la gestión local, Coordinado por la Organización Internacional para las Migraciones (OIM) y SISALVA (Universidad del Valle), Santiago de Cali, mayo-agosto.

Suárez Morales, Harvey Danilo (2001), “ Informe sobre los Sistemas de Información y Cifras sobre el Desplazamiento Forzado en Colombia", Documento de CODHES, Bogotá, Colombia.

Tovar, Hermes (1980), La formación social chibcha, Centro de Investigaciones y Educación, Universidad Nacional, Bogotá, Colombia.

Velez, Julian (2005), “Diagnóstico de los procesos de reconciliación en los municipios de Guática y Quinchía de Risaralda", Informe realizado para el proyecto de Justicia en Equidad, realizado con el apoyo técnico de la Universidad Nacional de Colombia. Bogotá. 